\section{Oleg Ptitsyn (1929-99)}

\section{Pioneer in protein folding}

Oleg Ptitsyn was one of those rare scientists who are prepared to advance new but unfashionable ideas, and pursue them until they either fall by the wayside or become generally accepted and move a subject forward. For nearly 30 years Ptitsyn advocated the concept of the 'molten globule' as a key intermediate in protein folding. This was a truly seminal idea - it breathed life into a seemingly intractable field, and as the concept developed it stimulated debate among a whole generation of experimentalists and theoreticians. Opinions still differ as to the semantic value of the terminology, and some members of the folding community use the words with evident anguish. But Ptitsyn's fundamental idea, that proteins can adopt compact structures without the close-packed side-chain interactions characteristic of native proteins, is now implicit in virtually every discussion of protein folding.

Oleg Ptitsyn was born in Leningrad in 1929 , and attended the university there as a student of physics. He had a doctorate by the age of 25 , and he became a prominent figure in the field of polymer theory through his work at the Institute of High Molecular Weight Compounds of the Academy of Sciences of the USSR in Leningrad. But by the 1960 s he had become fascinated by the ubiquitous polymers of the natural world, the proteins. As he later stated, "If a physicist wants to be useful in molecular biology he basically has the choice between establishing the structure of a biological macromolecule or trying to understand the physical basis of a biological process" (Trends Biochem. Sci. 20, 376-379; 1995). He had chosen the second way, adding "the main difficulty is finding a process that, while being biologically important, does not involve biochemical reactions, but rather is based purely on physical laws". He concluded that protein folding was the problem waiting to be solved. Nearly 40 years ago this was radical stuff indeed.

To begin research in this new area, Ptitsyn and others founded the Institute of Protein Research in the small scientific settlement of Pushchino, some 70 miles from Moscow. This institute became a world-famous centre for interdisciplinary research. And as a result of the general dispersal of Russian scientists over the past few years, its members have had an especially wide influence.

At Pushchino he allowed his students

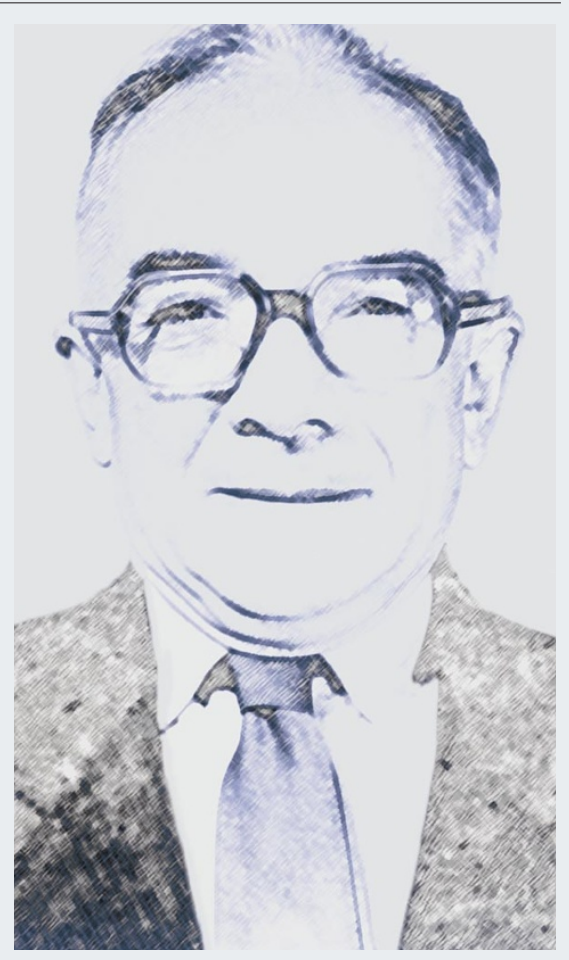

and younger colleagues a tremendous amount of intellectual freedom, while maintaining a supportive interest in their activities. It is said that every graduate student he supervised achieved a doctorate, a record of which he was justly proud.

In the early 1970s, Ptitsyn speculated that the protein-folding problem might be made much simpler if a polypeptide chain folds first into a rather flexible state with the native-like mutual positioning of helices and sheets, but without the intricate and detailed packing of the various side-chains found in a fully native protein. There was no experimental evidence for this proposal at the time, and the paper describing the idea passed rapidly through the offices of several journals before it was published ( Dokl. Akad. Nauk. SSSR 210, 1213-1215; 1973). Shortly afterwards, such evidence began to emerge from studies of protein denaturation in various laboratories. But Ptitsyn was keen to explore his idea himself, and he introduced the experimental strategy of applying a combination of physical methods to search for this new state of proteins. Progress was rapid, and the name 'molten globule' soon emerged (coined in fact by Akiyoshi Wada in Japan). The Ptitsyn laboratory subsequently made the major advance of identifying species in kinetic experiments that fitted his definition of a molten globule, hence relating this state to the mechanism of the folding process itself.
From 1992, Ptitsyn himself spent much of his time as a visiting scientist at the National Institutes of Health in Bethesda, although he also maintained an active group in Pushchino, and indeed collaborations around the globe. To the end of his life he continued to refine his ideas about the molten-globule concept, and to extend it to an ever-widening range of phenomena. He speculated, for example, that such a state could be important in translocation of proteins across membranes, and that its tendency to aggregate could make it a target for the molecular chaperones that help prevent aggregation in the cell. And he was one of the first to link protein misfolding events to disease.

In his later years, however, his greatest enthusiasm was reserved for the topic of molecular evolution. His view was that residues that are important for folding, as well as for function, should be conserved during evolution. By collaborating with other theoreticians and biologists, he obtained the beginnings of the case for this hypothesis. As he said: "Folding represents a bridge between the scientific principles on which the laws of physics are based and the results of the evolutionary pressures under which the character of biology has developed .... For many years we could only discuss this issue in very general terms ... now we are beginning to understand" (Curr. Opin. Struct. Biol. 9, 89-91; 1999). He was tasting at last the fruits of the seeds planted all those years ago in the physics laboratory at Leningrad.

Oleg Ptitsyn was a gentle, kindly person, whose diminutive and bustling figure was familiar around the conference and lecture halls of the world. His voice was unmistakable, and following his conversation was a challenge to even the best of linguists. But the effort was never wasted, and his words never contained any of the malice that can diminish the achievements of the greatest of men. He died on 22 March, just before he was due to give a lecture at the University of Warwick, during one of his frequent trips to Britain. It was as he would have wished. He died, as he had lived, earnestly engaged in the practice of science, and looking forward to intense discussions about his latest ideas.

Christopher M. Dobson and R. John Ellis

Christopher M. Dobson is at the Oxford Centre for Molecular Sciences, New Chemistry Laboratory, University of Oxford, South Parks Road, Oxford OX1 3QT, UK

e-mail: chris.dobson@chem.ox.ac.uk

R. John Ellis is in the Department of Biological Sciences, University of Warwick, Coventry CV4 7AL, UK

e-mail: je@dna.bio.warwick.ac.uk 